Proceedings

\title{
Embroidery Textile Moisture Sensor ${ }^{\dagger}$
}

\author{
Marc Martinez-Estrada, Bahareh Moradi, Raúl Fernández-Garcia * and Ignacio Gil \\ Department of Electronic Engineering, Universitat Politècnica de Catalunya, 08222 Terrassa, Spain; \\ marc.martinez-estrada@upc.edu (M.M.-E.); bahareh.moradi@upc.edu (B.M.); ignasi.gil@upc.edu (I.G.) \\ * Correspondence: raul.fernandez-garcia@upc.edu \\ + Presented at the Eurosensors 2018 Conference, Graz, Austria, 9-12 September 2018. \\ Published: 21 November 2018
}

\begin{abstract}
In this work, two embroidered textile moisture sensors are presented. The sensors are based on a capacitive interdigitated structure embroidered on a cotton substrate with an embroidery conductor yarn composed by $99 \%$ pure silver plated nylon yarn 140/17 dtex. In order to evaluate the sensor sensitivity, the impedance of the sensor has been measured by means of a LCR meter from $20 \mathrm{~Hz}$ to $20 \mathrm{kHz}$ on a climatic chamber with a sweep of the relative humidity from $25 \%$ to $65 \%$ at $20{ }^{\circ} \mathrm{C}$. The experimental results show a clear and controllable dependence of the sensor impedance with the relative humidity. Therefore, this dependence points out the usefulness of the proposed sensor to develop wearable applications on health and fitness scope.
\end{abstract}

Keywords: sensor; e-textile; embroidery; moisture

\section{Introduction}

Embroidery has been revealed as the most effective technique to implement wearable sensors. This fact is due to the availability of the manufacturing technology (industrial embroidery machines), efficient exploitation of the expensive specialized conductive threads and repeatability of geometries and layouts [1]. These wearable sensors are suitable for application fields such as health monitoring, physical training, emergency rescue service and law-enforcement [2]. In particular, the integration of flexible, lightweight and comfortable designs allows the deployment of wearable solutions [3]. In the last years, a great effort has been focused in designing new sensors included in garments for healthcare applications [4].

In this work, an embroidered textile sensor in order to measure the moisture is presented. A full characterization and modelling has been carried out. The remainder of the paper is organized as follows. Section 2 describes the Material and methods, the textile sensor layout and implementation as well as the measurement set-up. In Section 3 the experimental results are shown and discussed. Finally, in Section 4 the conclusions are summarized.

\section{Materials and Methods}

The proposed moisture sensor is based on a capacitive embroidered interdigitated structurewhose dimensions are depicted in Figure 1. A commercial Shieldex 117/17 dtex 2-ply has been chosen as a conductive yarn in order to embroider the interdigitated structures on a high hygroscope substrate. Specifically, a cotton substrate with a thickness (h) of $0.43 \mathrm{~mm}$ has been chosen. A Singer Futura XL-550 embroidery machine with a satin fill stitch pattern has been selected in order to achieve a homogeneous yarn distribution over the sensor surface. 


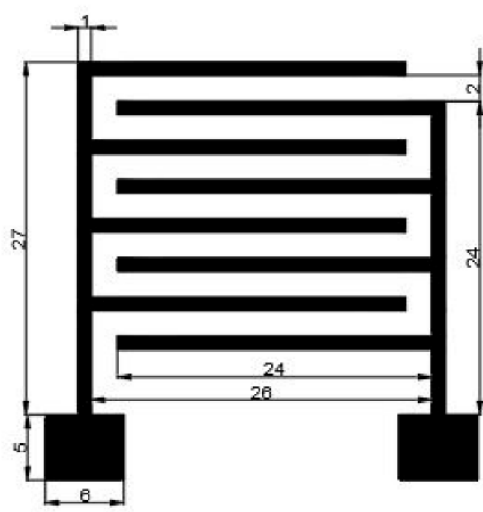

(a)

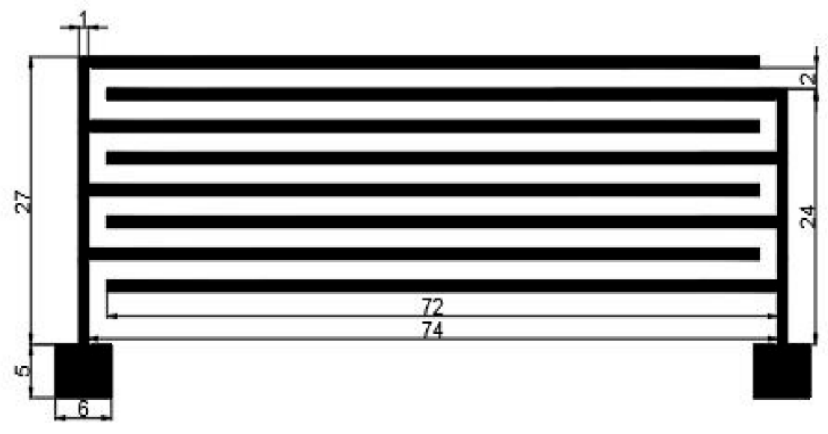

(b)

Figure 1. Layout and dimension detail of the proposed moisture sensor (in mm): (a) short sensor; (b) long sensor. The bottom squares correspond to the characterization pads.

In order to characterize the sensor behavior, the device has been tested ina CCK-25/48 Dycometal climatic chamber and the sensor impedance has been measured by means of an external Rohde \& Schwarz HM8118 LCR meter. An image of the experimental setup and embroidered short sensor are shown in Figure 2.

The sensors impedances have been measured from $20 \mathrm{~Hz}$ to $20 \mathrm{kHz}$ in a $25 \%$ to $65 \%$ of relative humidity environment, meanwhile the temperature has remained constant at $20^{\circ} \mathrm{C}$.

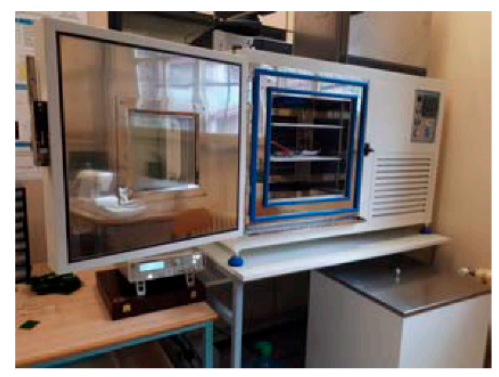

(a)

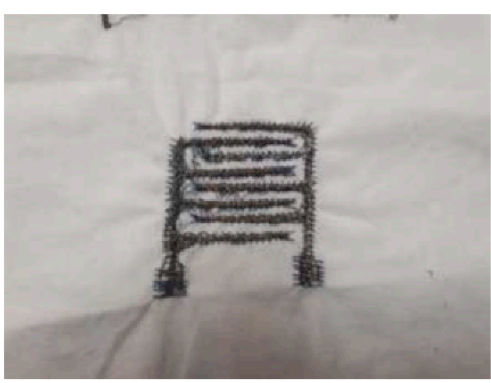

(b)

Figure 2. Image of the experimental setup. (a) climatic chamber, (b) embroidered short sensor

\section{Results and Discussion}

Figure 3 shows the measured sensor impedance when the moisture is swept from $25 \%$ to $65 \%$ for four different test frequencies. It is observed that the impedance module of sensor is reduced when the environmental moisture increases, which confirms the functionality of the proposed structure as a moisture sensor. The measured phase impedance of the sensor denotes that for low relative humidity the sensor has a capacitive behavior, as expected. Moreover, for higher relative humidity concentration the sensor tends to be resistive. The reason of this behavior is the hydrophilic property of the cotton. Indeed, when the relative humidity increases, the cotton substrate absorbs water and the electrical permittivity of the substrate increases. As a result, the impedance of the sensor is reduced.

Long and short sensor shows similar behavior with the relative moisture. However, as it is expected, the impedance of the longer sensor (Figure $3 b$ ) is lower than the impedance of the short device (Figure 3a). In particular, for the $20 \mathrm{~Hz}$ test signal, the short sensor impedance decreases from $1.1 \mathrm{G} \Omega$ to $20.4 \mathrm{M} \Omega$ when the moisture increases from $25 \%$ to $65 \%$, whereas, for long sensor device it decreases from $0.83 \mathrm{G} \Omega 1012.5 \mathrm{M} \Omega$ for the same moisture range. 

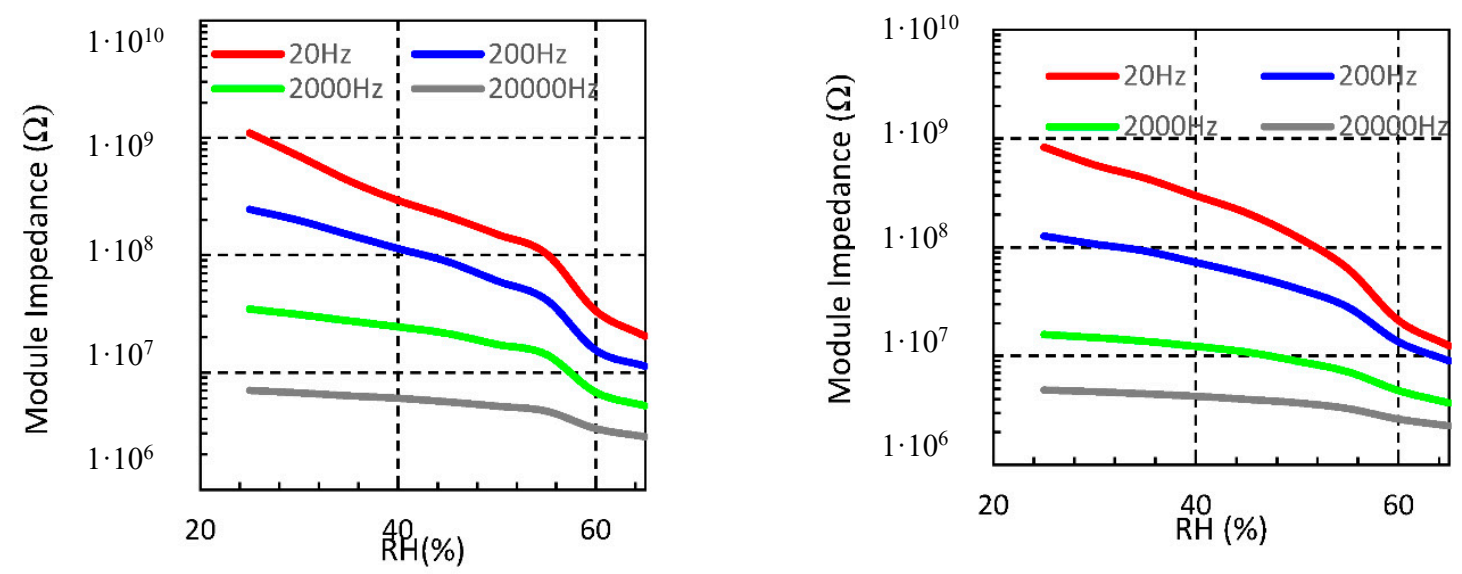

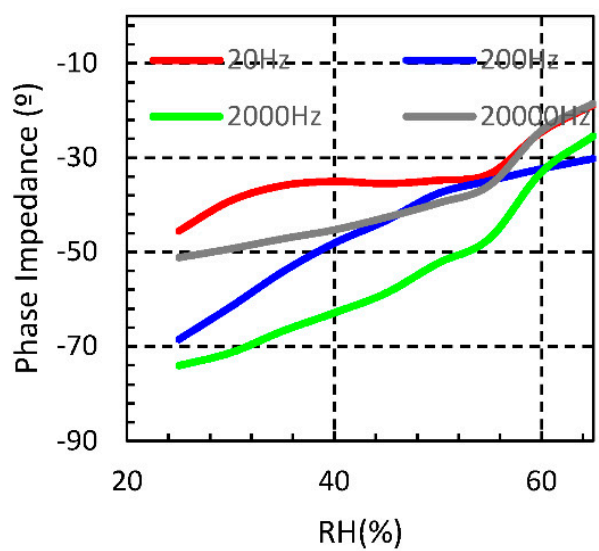

a) Short sensor impedance.

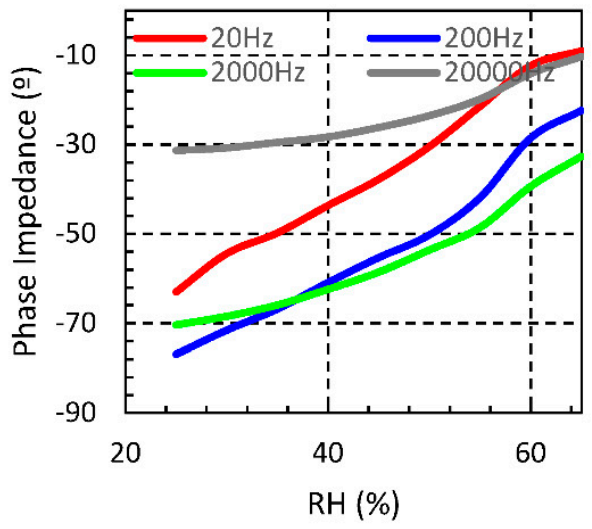

b) Long sensor impedance.

Figure 3. Measured sensors impedance from $25 \%$ to $65 \% \mathrm{RH}$ at different frequencies $\left(\mathrm{T}=20^{\circ} \mathrm{C}\right)$.

It should be noticed that the obtained impedance values at the low frequency range are too high in order to develop a portable device based on the proposed sensor. These wearable devices are typically based on a single integrated circuit, such as the AD5933 impedance converter [5] (Texas Instruments, Dallas, USA). Nevertheless, using the proposed sensor in the range of $\mathrm{kHz}$ allows obtaining impedances in the range of a $\mathrm{M} \Omega$. In these cases, the impedance values that can be measured with this integrated circuits.

If we focus on the electronic performance according to the behavior of the proposed sensors at $2 \mathrm{kHz}$, they can be modelled as a RC parallel lumped model (Figure $4 \mathrm{~b}$ ), where the R and C values are moisture dependent.

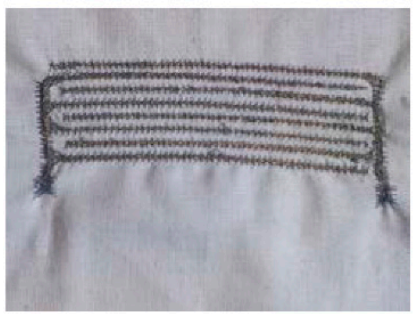

a)

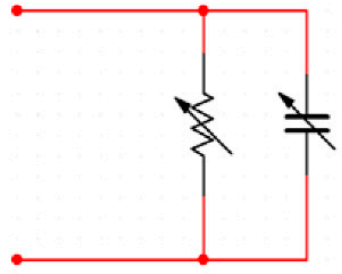

b)

Figure 4. Proposed long sensor (a) physical implementation (b) Electrical model.

In Figure 5 the $\mathrm{R}$ and $\mathrm{C}$ dependence from $25 \%$ to $65 \% \mathrm{RH}$ at $2 \mathrm{kHz}$ is shown for both short and long sensor. It can be observed than when the moisture level increases the capacitance is increased, whereas the resistance is reduced in both sensors. It should be pointed out that both sensors show a 
similar trend with the moisture impact, with a clear sensitivity change around 55\% RH. Despite, the short sensor has a lower capacitance and higher resistance value, due to his physical dimensions. In fact, the capacitance increases from $1.45 \mathrm{pF}$ to $8.84 \mathrm{pF}$ for the short sensor and from $3.13 \mathrm{pF}$ to 19.40 $\mathrm{pF}$ for the long sensor. Meanwhile, the resistance decreases from $4.42 \mathrm{M} \Omega$ to $2.68 \mathrm{M} \Omega$ and from 4.17 $\mathrm{M} \Omega$ to $2.26 \mathrm{M} \Omega$ for the short and long sensor, respectively.

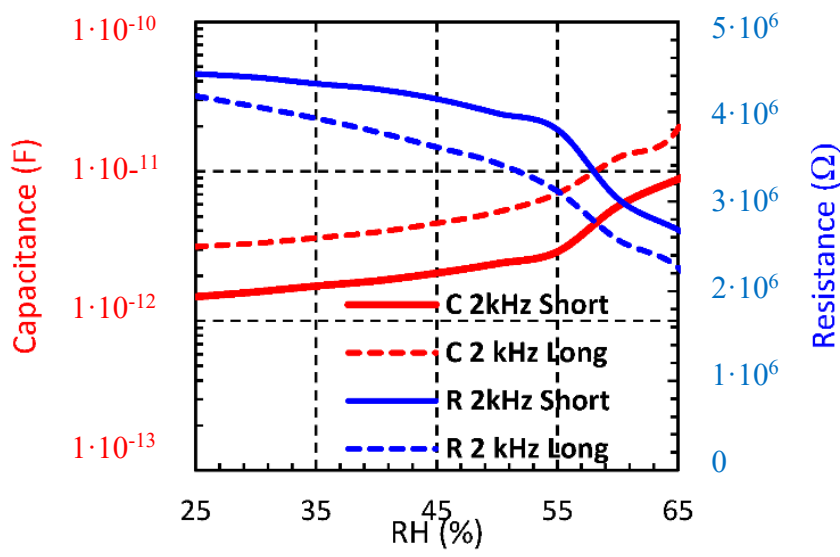

Figure 5. Measured sensors impedance from $25 \%$ to $65 \%$ RH at different frequencies.

\section{Conclusions}

In this work two interdigitated embroidered textile sensors have been proposed and characterized. The sensors have been embroidered over a cotton substrate with a commercial Shieldex 117/17 dtex 2 yarn. The measured results show that the proposed sensors can be modelled by means of a RC parallel lumped circuit, where the $\mathrm{R}$ and $\mathrm{C}$ value are moisture dependent. These preliminary results demonstrate experimentally the usefulness of the proposed sensors at the $\mathrm{kHz}$ range to develop wearable application over textiles materials for health and fitness applications, such as the sweating measurement.

Acknowledgments: This work was supported by the Spanish-MINECO Project TEC2016-79465-R.

Conflicts of Interest: The authors declare no conflict of interest.

\section{References}

1. Castano, L.M.; Flatau, A.B. Smart Fabric Sensors and E-Textile Technologies: A Review. Smart Mater. Struct. 2014, 23, 053001.

2. Tartare, G.; Zeng, X.; Koehl, L. Development of a Wearable System for Monitoring the Firefighter's Physiological State. In Proceedings of the 2018 IEEE Industrial Cyber-Physical Systems (ICPS), St. Petersburg, Russia, 15-18 May 2018; pp. 561-566.

3. Saenz-Cogollo, J.; Pau, M.; Fraboni, B.; Bonfiglio, A. Pressure Mapping Mat for Tele-Home Care Applications. Sensors 2016, 16, 365.

4. Bouwstra, S.; Chen, W.; Feijs, L.; Oetomo, S.B. Smart Jacket Design for Neonatal Monitoring with Wearable Sensors. In Proceedings of the 2009 Sixth International Workshop on Wearable and Implantable Body Sensor Networks, Berkeley, CA, USA, 3-5 June 2009; pp. 162-167.

5. Devices, A. AD5933 1 MSPS, 12-Bit Impedance Converter, Network Analyzer. Available online: http://www.analog.com/media/en/technical-documentation/data-sheets/AD5933.pdf (accessed on 15 May 2018)

(C) 2018 by the authors. Licensee MDPI, Basel, Switzerland. This article is an open access article distributed under the terms and conditions of the Creative Commons Attribution (CC BY) license (http://creativecommons.org/licenses/by/4.0/). 